\title{
Performance and Challenges in Utilizing Non-Intrusive Sensors for Traffic Data Collection
}

\author{
Xin $\mathrm{Yu}^{1}$, Panos D. Prevedouros ${ }^{2}$ \\ ${ }^{1}$ Stantec Inc., Edmonton, Canada \\ ${ }^{2}$ Department of Civil and Environmental Engineering, University of Hawaii at Manoa, Honolulu, USA \\ Email: alyx.yu@stantec.com,pdp@hawaii.edu
}

Received February 22, 2013; revised March 25, 2013; accepted April 2, 2013

Copyright (C) 2013 Xin Yu, Panos D. Prevedouros. This is an open access article distributed under the Creative Commons Attribution License, which permits unrestricted use, distribution, and reproduction in any medium, provided the original work is properly cited.

\begin{abstract}
Extensive field tests of non-intrusive sensors for traffic volume, speed and classification detection were conducted under a variety of traffic composition and road width conditions. The accuracy challenges of utilizing non-intrusive sensors for traffic data collection were studied. Both fixed and portable sensors with infrared, microwave and image recognition technologies were tested. Most sensors obtained accurate or fairly accurate measurements of volume and speed, but vehicle classification counts were problematic even when classes were reduced to 3 to 5 compared to FHWA's 13-class standard scheme.
\end{abstract}

Keywords: Non-Intrusive; Traffic Sensor; Classification

\section{Introduction}

Transportation agencies are responsible for establishing and maintaining a traffic monitoring and data collection system that meets the requirements of the Traffic Monitoring Guide [1] of Federal Highway Administration (FHWA) and the data needed for local and regional traffic analysis. Traffic data including vehicle speed, volume and classification needed for pavement design, transportation demand forecasting, implementation of intelligent transportation systems and development of traffic facilities and infrastructure typically come from sensors in, on or over roads.

Vehicle sensing technologies used in traffic monitoring and data collection are intrusive or non-intrusive based on their impacts on the road pavement during installation, use and maintenance. Intrusive traffic detectors are required to be installed directly on the pavement surface, in cuts or holes of the road surface, by tunneling under the surface or anchoring directly to the pavement surface. Non-intrusive traffic detectors are mounted overhead ("overlane" sensor) or on the side of the roadway ("sidefire" sensor).

Traditional intrusive sensors include the inductive loop, magnetic, magnetometer, pneumatic and pressure sensitive; these are widely employed in the US. The use of

*This paper is a product of research supported by the Hawaii State DOT and the FHWA. The support of the sponsors is gratefully acknowledged. traditional sensors embedded in roads for collecting data is necessary but not sufficient because of their limited coverage and expensive implementation and maintenance, especially in busy urban areas. In addition, traffic delay, accident risk and crew safety risk increase during the temporary closure of traffic lanes for installation and maintenance of intrusive traffic detectors.

Currently, the traditional intrusive technologies are more widely applied in traffic data collection than nonintrusive technologies in part because of institutional inertia and know-how. However, newer non-intrusive technologies are rapidly evolving and non-intrusive sensors are becoming more reliable and easier to deploy and use. Compared with intrusive sensors, the advantage of non-intrusive sensors is to minimize interference with traffic flow and reduction of safety risk. In addition, in bridges and tunnels where pavement cutting and boring are undesirable, non-intrusive sensors present a suitable alternative. The application of non-intrusive detectors is accelerating: Among 46 responding agencies, two thirds use non-intrusive sensors for traffic counts according to a nationwide survey conducted by Colorado Department of Transportation (DOT) [2].

Non-intrusive technologies include infrared, lightsensitive, microwave radar, sound-sensitive, ultrasonic, and video image processing (VIP) sensors. They are able to detect the heavy traffic of urban arterials and freeways 
exhibiting large fluctuations in traffic flow speed and density. Recent studies found that the current non-intrusive sensors improve the detection of volume and speed data as well as capability of potable classification data collection [3], is capable of replacing loops or intrusive sensors for traffic signal system [4], and is safe and convenient for deployment and maintenance on urban highway and freeway [5,6].

The difficulties and limitations associated with traditional data collection methods such as sensitivity to environmental conditions, traffic flow conditions and roadway geometrics may also challenge the accuracy, reliability and functionality of non-intrusive traffic detectors. The use of non-intrusive sensors for traffic data collection requires an investigation to discover the proper combination of local, geometric, operational and sensor features that result in an accurate and robust traffic monitoring system.

This study contributed to the application and challenges of portable and non-intrusive traffic sensors for temporary or permanent traffic data collection. Various non-intrusive technologies were tested and evaluated in a variety of traffic and environmental conditions at urban and rural arterial, highway and freeway test sites.

\section{Investigation of Non-Intrusive Technologies}

Non-intrusive traffic detectors provide volume and speed detection but most of them can only classify vehicles based on their length and only into 3 to 8 user-selected length bins. A small subset of non-intrusive sensing technologies such as infrared laser have the capability of differentiating among types of vehicles based on the number of axles which is the standard 13 class scheme required by FHWA [1]. FHWA encourages transportation agencies to evaluate sensor technologies based on the data quality they expect, the traffic information they require, the location where they plan to use the equipment and the funds available for sensor purchase and operation [3].

\subsection{Selection of Non-Intrusive Technologies}

When selecting non-intrusive vehicle detectors, two major factors need be taken into account before conducting research. First, any sensors that could be used for collecting traffic data must be able to satisfy the data requirements (e.g., supported data types, vehicle classification methods, and precision of detection based on the manufacturer specifications or claims). Second, when choosing a particular type of vehicle detector for a specific local use, two more questions need to be answered: (a) What is the level of skill required for installation and maintenance of the devices? (b) What are the geometric and traffic characteristics of the deployment sites? The answers to these questions are essential for optimizing device selection.

If the traffic sensors are tested for permanent deployment, then the year-long characteristics of climate and environment at the deployment sites should be considered. Some sensors are more sensitive to environmental variation (e.g., temperature, humidity and precipitation) or require more stringent operating conditions than others. A variety of environmental variables can affect the accuracy of non-intrusive technologies [7]. For example, if a device is proposed to be installed at a site where the intensity of illumination is insufficient and the shadoweffect of surrounding obstacles (e.g. trees, bridges or buildings) is pervasive, then an image processing VIP sensor will be least appropriate.

Traffic data collection can be done with a multitude of sensing technologies. Detectors should after reviewing different technologies rather than narrowing the detector selection to only one particular technology. Given the pace of sensor technology improvement, evaluating and upgrading non-intrusive sensors every ten years or so is prudent [3], so sensor replacements can take advantage of improved accuracy and possibly lower cost per lane of coverage.

This study selected several non-intrusive traffic sensors which are used by transportation agencies and feature mature sensing technologies: AutoscopeRackVision (Autoscope) with image processing technology, Infra-red Traffic Logger (TIRTL) with active infrared technology, Remote Traffic Microwave Sensor (RTMS), SmartSensor 105 (SS105) and SmartSensor HD (SSHD) with microwave radar technology, and SmarTek's Acoustic Sensor (SAS-1) with passive acoustic technology were tested.

\subsection{Sensor Deployment and Tests}

The purpose of our tests was to investigate the ability of specific non-intrusive detectors for collecting traffic data and discover the potential challenges associated with deployment and use. There are five major steps in these tests:

1) Selection of field test sites: The test locations were determined in coordination with the Hawaii Department of Transportation (HDOT). Field trips were taken to assess the geographic environment, weather, and traffic conditions of the candidate test sites. Earlier research had identified appropriate locations for sensor deployment on Oahu [8].

2) Selection of equipment for field tests: The test equipment was selected from available commercial nonintrusive sensors. Six types of sensors featuring four types of non-intrusive sensor technologies were fieldtested. 
3) Sensor deployment and calibration: Project researchers, with assistance from manufacturers and HDOT, installed and calibrated sensors at the selected sites. A careful short term data collection (usually 15 minutes) and verification with manual direct observation was conducted to ensure proper installation and calibration. This was achieved with the laptop computer interface of each sensor.

4) Technical training of sensor operation: Technical training on device management and application of bundled software was conducted by some of the manufacturer technicians. Remote access and control of sensors was established, which enabled researchers to observe real time status of operation via the Internet.

5) Data collection and evaluation: The data collection was conducted in two parts: independent field tests and integrated field tests. During the independent field tests, one of the selected was deployed at different locations and was tested for reliability and accuracy of traffic counts. The traffic data from the sensor were compared with manual classifications taken from simultaneous video recordings. Integrated field tests included simultaneous collection of traffic data at the same location using multiple sensors. In addition to recording synchronous video to identify misclassification errors of each sensor, the peak and non-peak hour data collected by two or more sensors were compared with each other.

Tests and technology demonstration deployments were conducted on arterials, freeways and highways in urban, rural and industrial areas in and around Honolulu between February 2008 and May 2011. The sensor deployments and objectives are summarized in Table $\mathbf{1}$ including the locations and devices involved in independent and integrated sensor tests.

Table 1. Summary of sensor tests and deployments.

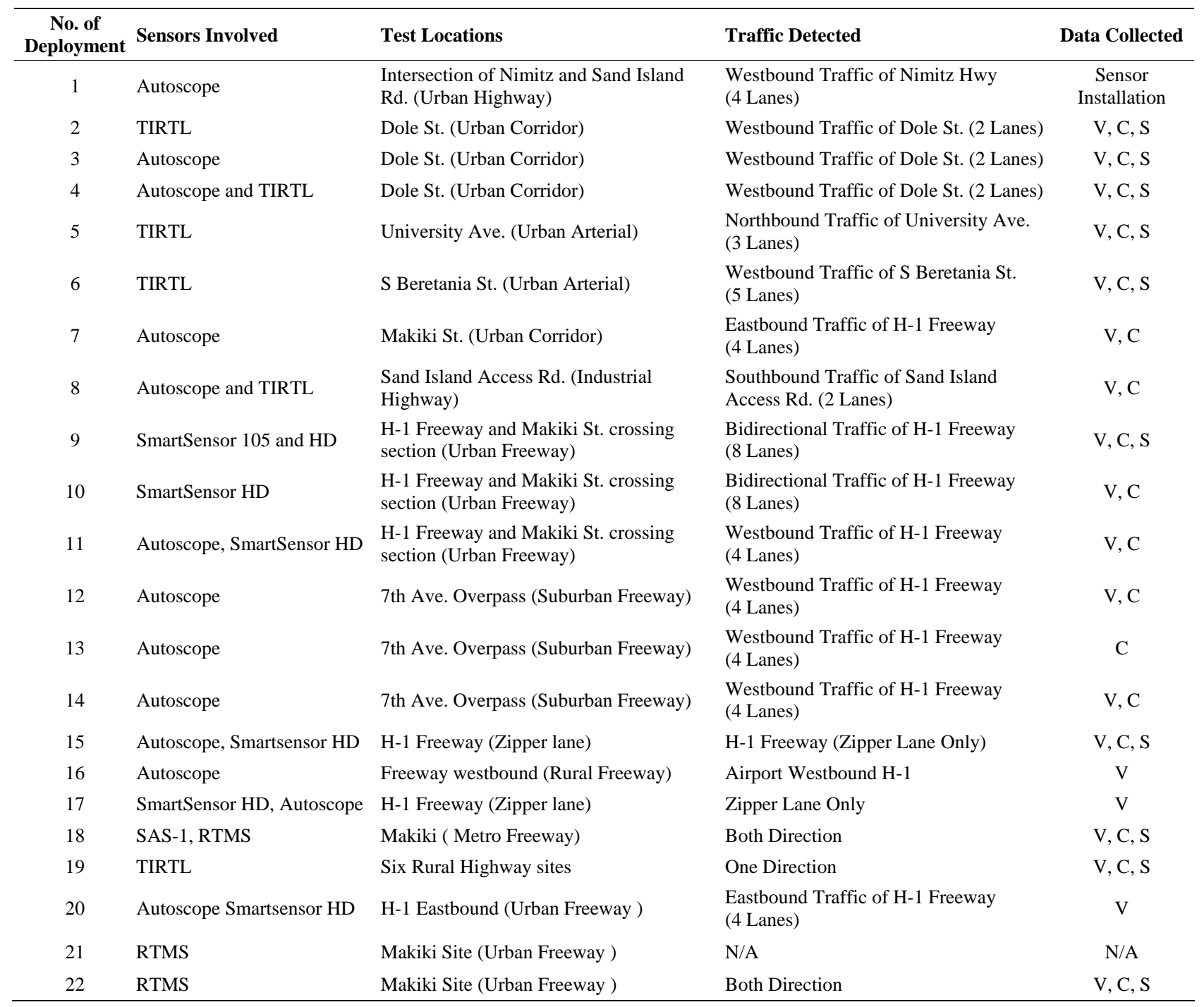

$\mathrm{V}=$ volume; $\mathrm{C}$ = classification; $\mathrm{S}=$ speed. 


\section{Test Results and Challenges}

\subsection{Summary of Test Results}

The test results were compiled from field demonstrations and included adverse traffic and environmental conditions. The accuracy percentages for volume, speed and classification measurements by using those sensing technologies in this study are summarized in Table 2. This section only briefly presents and summarizes the test results on non-intrusive sensor tests. A more expansive analysis and discussion can be found in [9-11].

The accuracy of volume and classification detection utilizing VIP technology is $98 \%$ and $92 \%$ in daytime, but it sharply degrades to $82 \%$ and $64 \%$ at night. The significant degradation in volume and classification count accuracy can also be observed at the urban freeway test sites (i.e., deployment 16). VIP technology is adversely affected by the strong shadow of roadside structures or trees. The shadow of tall vehicles in one lane spilled onto the neighboring lane and triggered a false detection. Drops of rain obstructed the view of the camera affecting the sensor's measurements. VIP sensor performance for speed detection was insensitive to the effects of shadow, level of illumination and rainfall. Traffic flow density didn't affect the volume-only counts significantly, but heavy traffic decreases the accuracy of vehicle classification.

The infrared laser sensor with a detection range up to $200 \mathrm{ft}$. provided good accuracy volume, speed and classification (axle-based), and especially for the detection of heavy vehicles. The infrared laser sensor also worked well under different traffic conditions. If the equipment enclosure is completely sealed, this study observed no impact on the performance during rainy weather. However, the Traffic Detector Handbook [9] suggests that active infrared sensors may be affected by fog, rainstorm or snow when visibility is less than $6 \mathrm{~m}$. In addition, because vehicle class is determined by detecting the number of vehicle axles and spacing, if the infrared beams are blocked by slow moving or stopped vehicles under congested flow, then vehicle detection will cease and the passing vehicles during the blockage period will be missed or axles will be missed resulting in misclassifications.

Table 2. Summary of sensor test results.

\begin{tabular}{|c|c|c|c|c|c|c|}
\hline Tested Technologies & No. of Deployment & & Accurac & & Condition & \\
\hline \multirow{8}{*}{$\begin{array}{l}\text { Video Image } \\
\text { Processing } \\
\text { (Autoscope) }\end{array}$} & \multirow{4}{*}{$2,3,4$} & Vol. & Spd. & Cls. & Traffic & Weather \\
\hline & & $98 \%$ & $94 \%$ & $92 \%$ & Moderate Mixed Traffic & Daytime \\
\hline & & $82 \%$ & $94 \%$ & $64 \%$ & Moderate Mixed Traffic & Nighttime \\
\hline & & $84 \%$ & $93 \%$ & $78 \%$ & Moderate Mixed Traffic & Shadow \\
\hline & $7,10,11$ & $91 \%$ & & $73 \%$ & Heavy Mixed Traffic & Clear \\
\hline & $12,13,14$ & $93 \%$ & & $87 \%$ & Light Mixed Traffic & Clear \\
\hline & 15,17 & $97 \%$ & $95 \%$ & $90 \%$ & Morning Peak, Single Divided Lane & Clear \\
\hline & 16 & $98 \%$ & & & 24 Hours Counts & Daytime \\
\hline \multirow{4}{*}{$\begin{array}{l}\text { Infrared Laser } \\
\text { (TIRTL) }\end{array}$} & \multirow{2}{*}{2,4} & $92 \%$ & $94 \%$ & $96 \%$ & Moderate Mixed Traffic & Daytime \\
\hline & & $93 \%$ & $94 \%$ & $96 \%$ & Moderate Mixed Traffic & Nighttime \\
\hline & 8 & $94 \%$ & & $95 \%$ & Moderate Truck Traffic & Rainy \\
\hline & 19 & $91 \%$ & & $97 \%$ & Light Mixed Traffic & Clear \\
\hline \multirow{4}{*}{$\begin{array}{l}\text { Microwave Radar } \\
\text { (SSHD) }\end{array}$} & \multirow{2}{*}{$9,10,11$} & $91 \%$ & & $81 \%$ & Heavy but Non-Congested Traffic & Light Rain \\
\hline & & $89 \%$ & & $80 \%$ & Congested Traffic & Clear \\
\hline & \multirow{2}{*}{15,17} & $93 \%$ & $95 \%$ & & Morning Peak, Single Divided Lane & Clear \\
\hline & & $87 \%$ & & & 24 Hours Counts & Clear \\
\hline $\begin{array}{l}\text { Microwave Radar } \\
\text { (SS105) }\end{array}$ & 9 & $89 \%$ & $94 \%$ & & Heavy but Non-Congested Traffic & Light Rain \\
\hline \multirow{2}{*}{$\begin{array}{l}\text { Microwave Radar } \\
\text { (RTMS) }\end{array}$} & \multirow{2}{*}{21,22} & $95 \%$ & $91 \%$ & $77 \%$ & Heavy but Non-Congested Traffic & Clear \\
\hline & & $91 \%$ & & $63 \%$ & Congested Traffic & Clear \\
\hline $\begin{array}{l}\text { Passive Acoustic } \\
\text { (SAS-1) }\end{array}$ & 18 & $97 \%$ & $90 \%$ & $61 \%$ & Moderate Mixed Traffic & Clear \\
\hline
\end{tabular}


The microwave radar sensor with a detection range of eight lanes is the most widely used non-intrusive traffic detector and is relatively inexpensive and two models of traffic detectors with microwave radar technologies are tested in this study, an older 2004 model and a newer "high density" 2009 model. Based on the test results, this sensor can provide $90 \%$ accuracy in volume and $75 \%$ in classification detections, on average, under optimal conditions, which is inferior to the performance of VIP and laser sensor under similar conditions. Also there is a slight degradation of performance under congested traffic but no degradation under adverse weather. Double counting becomes frequent in high density (stop-and-go) flow, which occurs when the detection of a single vehicle is stopped and then resumed, thus registering as two vehicles.

The passive acoustic sensor is a side mounted sensor with a detection range of up to five lanes. It can collect traffic counts with three levels of classification based on vehicle size (small, medium and large). The acoustic sensor provided accurate volume accounts and adequate speed detection but its detection of vehicle class is not satisfactory. Further research on acoustic sensor performance was terminated because of our focus on classification.

\subsection{Challenges in Utilizing Non-Intrusive Technologies}

Non-intrusive technologies have become increasingly able to detect heavy traffic of urban arterials and freeways and the traffic delay and safety risk caused by sensor deployment are minimal. However, this research found that the application of non-intrusive technologies also presents difficulties and limitations in traffic data collection as follows.

1) Restrictive installation requirements: The process of installation and maintenance of non-intrusive sensors typically poses minimal impacts on road traffic and is not associated with severe safety concerns. But the deployments of sensors usually require specific roadway geometric, facing angle adjustment and mounted location. For example, the overhead deployment of VIP sensor must have a mounted height of 12 to $15 \mathrm{~m}$ for the video camera [12]. Camera mounted at $9 \mathrm{~m}$ or lower would generate significant errors at the traffic detection based on the test results in this study and by Texas DOT [12]. The video image of large vehicles can spill-out and block the entire observation zone if the camera height is not adequate. However, typical light and utility poles along roadways rarely exceed $10 \mathrm{~m}$ height, which means that very expensive custom installations are likely required for VIP. Also VIP sensors requiring large vertical and horizontal clearances are not suitable for tunnels.
Microwave and acoustic sensors have a typical lateral offset requirement of 5 to $8 \mathrm{~m}$. Finding poles $8 \mathrm{~m}$ away from the nearest lane of a roadway may be difficult, thus, again a custom installation may be necessary. This raises costs substantially. However, there is a solution to this problem for 4 to 8 lane wide arterial streets and highways with a relatively low median or no median. Assuming that the road runs in an East-West direction and there are poles, say at a distance of $1 \mathrm{~m}$ from the near lane on both sides, the East side sensor is installed to monitor westbound traffic flow and the West side sensor is installed to monitor Eastbound traffic flow. This solution provides a 2 to 3 lane lateral offset and may work well for some microwave sensors subject to field test and verification.

The deployment of TIRTL also requires flat pavement with a minimal cross-sectional crown. Road surfaces with pronounced crowns, slopes or protruding objects (median separator or speed bump) may lead to large errors or render many desirable cross-sections unsuitable for deployment.

2) Environmental Sensitivity: The accuracy of data collection by the VIP sensor is sensitive to reductions of illumination, shadows and weather variations. Rainfall normally has limited effect on infrared sensor but dense fog, heavy precipitation or snow could cause traffic to be undercounted. Relatively few highway locations may be suitable for permanent deployment and continuous data collection, but VIP disadvantages become advantages when it comes to tunnels where light conditions are far more predictable and precipitation is absent. Most modern cameras have several cameras installed for safety monitoring so suitable VIP sensors and customized algorithms for lower heights and level of illumination may provide adequate data collection [13].

3) Relatively High Cost: The availability of non-intrusive data collection equipment may justify the conversion of traditional data collection sites. Compared to inductive loops, the cost of several advanced non-intrusive sensors that provide detailed traffic flow data measurements is usually higher. High costs impede widespread distribution and limit the application of non-intrusive in gathering traffic information. For example, in this study, the most expensive sensor, infrared laser sensor, provides the best performance in monitoring traffic flow. The relatively inexpensive non-intrusive microwave or acoustic array sensors did not provide satisfactory classification and the accuracy of volume and speed counts were relatively inferior to other non-intrusive sensors tested in this study.

Despite these issues, non-intrusive loop detectors have the advantage of being accurate and convenient when installed, calibrated and operated properly. The high cost and relative sensitivity to external conditions is largely due to the facts that the application of non-intrusive tech- 
nology in traffic monitoring is improving and has had fewer than 20 years of development. Also, limited studies and investigations have been conducted to analyze the strengths and weakness of these sensors and transportation professionals are not very familiar with the operational and maintenance needs of these newer data collection methods. Indeed institutional and in-house technical knowledge, expertise and funds for re-training may be the most significant barriers for the extensive adoption of non-intrusive, advanced sensors.

\section{Conclusion}

Volume, speed and classification results as well as the challenges of using non-intrusive technology sensors for traffic data collection was presented based on a large number of field deployments and tests under various traffic and environmental conditions. The main findings drawn from the research are that the VIP sensor can provide reliable traffic volume, speed and classification counts under non-congested traffic conditions and optimal weather condition, once the sensor is optimally calibrated and the video camera is properly mounted. The expensive infrared laser sensor is superior to other technologies in traffic detection and lack of sensitivity to adverse conditions. Microwave radar sensors and the acoustic sensor provided adequately accurate traffic volume and speed detection but their performance in vehicle classification was not adequate.

\section{REFERENCES}

[1] Office of Highway Policy Information, "Traffic Monitoring Guide,” US Department of Transportation/Federal Highway Administration, Washington DC, 2001.

[2] E. Stolz, “State Department of Transportation's (DOT’s) Travel Monitoring Survey Results Report," Colorado DOT, Division of Transportation Development, Denver, 2007.

[3] L. A. Klein, “Sensing Technologies and Data Require- ments for ITS,” Artech House Publishers, Norwood, 2001.

[4] D. Middleton, H. Charara and R. Longmire, "Alternative Vehicle Detection Technologies for Traffic Signal Systems: Technical Report,” Texas Transportation Institute, The Texas A\&M University System, College Station, 2009.

[5] X. Yu, "Evaluation of Non-Intrusive Sensors for Vehicle Classification on Freeways," Proceedings of 2nd International Symposium on Freeway and Tollway Operations, Honolulu, 21-24 June 2009, 13p.

[6] B. W. Grone, "An Evaluation of Non-Intrusive Traffic Detectors at the Ntc/Ndor Detector Test Bed,” Master Thesis, University of Nebraska, Lincoln, 2012.

[7] E. Minge, "Evaluation of Non-Intrusive Technologies for Traic Detection,” Research Project, Final Report \#201036, Minnesota Department of Transportation, Saint Paul, 2010.

[8] P. D. Prevedouros, "Detector Installation and Tests: Final Report,” Project Report, University of Hawaii at Manoa, Manoa, 2004.

[9] US Department of Transportation/Federal Highway Administration, "Traffic Detector Handbook," 3rd Edition, US Department of Transportation/Federal Highway Administration, Washington DC, 2006.

[10] X. Yu, P. D. Prevedouros and G. Sulijoadikusumo, "Evaluation of Autoscope, Smart Sensor HD and TIRTL for Vehicle Classification,” Transportation Research Record, Transportation Research Board, Washington DC, 2010.

[11] X. Yu, G. Sulijoadikusumo, H. L. Li and P. Prevedouros, "Reliability of Automatic Traffic Monitoring with NonIntrusive Sensors," Proceedings of the 11th International Conference of Chinese Transportation Professionals, Nanjing, 14-17 August 2011, pp. 4157-4169. doi:10.1061/41186(421)414

[12] Texas Transportation Institute, "Assessing Vehicle Detection Utilization Videp Image Processing Technology," Texas Department of Transportation, Austin, 1996.

[13] P. D. Prevedouros, J. Ji, K. Papandreou, P. Kopelias and V. Vegiri, "Video Incident Detection Tests in Freeway Tunnels," Transportation Research Record, Transportation Research Board, Washington DC, 2006 